Pacific Journal of Mathematics

POSITIVE-DEFINITE DISTRIBUTIONS AND INTERTWINING 


\title{
POSITIVE-DEFINITE DISTRIBUTIONS AND INTERTWINING OPERATORS
}

\author{
RoE Goodman
}

\begin{abstract}
An example is given of a positive-definite measure $\mu$ on the group $\mathrm{SL}(2, R)$ which is extremal in the cone of positivedefinite measures, but the corresponding unitary representation $L^{\mu}$ is reducible. By considering positive-definite distributions this anomaly disappears, and for an arbitrary Lie group $G$ and positive-definite distribution $\mu$ on $G$ a bijection is established between positive-definite distributions on $G$ bounded by $\mu$ and positive-definite intertwining operators for the representation $L^{\mu}$. As an application, cyclic vectors for $L^{\mu}$ are obtained by a simple explicit construction.
\end{abstract}

Introduction. The use of positive-definiteness as a tool in abstract harmonic analysis has a long history, the most striking early instance being the Gelfand-Raikov proof via positive-definite functions of the completeness of the set of irreducible unitary representations of a locally compact group [5]. More recently, it was observed by R. J. Blattner [1] that the systematic use of positive-definite measures gives very simple proofs of the basic properties of induced representations, and the cone of positive-definite measures on a group was subsequently studied by Effros and Hahn [4].

The purpose of this paper is two-fold. First, we give an example to show that positive-definite measures do not suffice for the study of intertwining operators and irreducibility of induced representations, despite the claim to the contrary in [4]. Specifically, we exhibit a positive-definite measure $\mu$ on $G=\mathrm{SL}(2, R)$ such that $\mu$ lies on an extremal ray in the cone of positive-definite measures on $G$, but the associated unitary representation $L^{\mu}$ is reducible, contradicting Lemma 4.16 of [4].

Our second aim is to show that when $G$ is any Lie group, then the correspondence between intertwining operators and positive functionals on $G$ asserted by Effros and Hahn does hold, provided one deals throughout with positive-definite distributions instead of just measures. The essential point is the validity of the Schwartz Kernel Theorem for the space $C_{0}^{\infty}(G)$, together with a result of Bruhat [3] about distributions on $G \times G$, invariant under the diagonal action of $G$. Using this correspondence, we obtain cyclic vectors for representations defined by positive-definite distributions, using a modification of the construction in [7]. (The proof of cyclicity given in [7] is invalid, since it assumes the existence of a measure on $G$ corresponding to 
an arbitrary intertwining operator. Cf. [6] for a proof of cyclicity using von Neumann algebra techniques.)

1. Notation and statement of theorems. Let $G$ be a Lie group, and denote by $\mathscr{D}(G)$ the space $C_{0}^{\infty}(G)$ with the usual inductive limit topology [10]. Fix a left Haar measure $d x$ on $G$; then $d(x y)=\Delta_{G}(y) d x$, where $\Delta_{G}$ is the modular function for $G$. If $\phi \in \mathscr{D}(G)$, define $\phi^{*}(x)=$ $\overline{\phi\left(x^{-1}\right) \Delta_{G}}(x)^{-1}$. Denote by $\mathscr{D}^{\prime}(G)$ the space of Schwartz distributions on $G$. A distribution $\alpha$ is positive-definite if $\alpha\left(\phi^{*} * \phi\right) \geqq 0$ for all $\phi \in \mathscr{D}(G)$, where convolution is defined as usual by

$$
(\psi * \phi)(x)=\int_{G} \psi(y) \phi\left(y^{-1} x\right) d y .
$$

If $\alpha$ and $\beta$ are distributions, say that $\alpha \ll \beta$ if $\beta-\alpha$ is positive-definite.

Given a positive-definite distribution $\mu$, one obtains a unitary representation $L^{\mu}$ of $G$ by a standard construction: Let $L_{y} \phi(x)=$ $\phi\left(y^{-1} x\right)$ be the left action of $G$ on $\mathscr{D}(G)$. Then $\left(L_{y} \phi\right)^{*} *\left(L_{y} \psi\right)=\phi^{*} * \psi$, so the semi-definite inner product $\mu\left(\phi^{*} * \psi\right)$ is invariant under left translations. Define $I_{\mu}=\left\{\phi \in \mathscr{D}(G): \mu\left(\phi^{*} * \phi\right)=0\right\}$. The quotient space $\mathscr{D}_{\mu}=\mathscr{D}(G) / I_{u}$ is then a pre-Hilbert space with inner product $(\tilde{\psi}, \tilde{\phi})_{\mu}=$ $\mu\left(\phi^{*} * \psi\right)$, where $\phi \rightarrow \tilde{\phi}$ is the natural mapping of $\mathscr{D}(G)$ onto $\mathscr{D}_{\mu}$. Let $\mathscr{H}_{\mu}$ be the completion of $\mathscr{D}_{\mu}$. The operators $L_{y}$ pass to the quotient to give a strongly continuous unitary representation $y \rightarrow L_{y}^{\mu}$ of $G$ on $\mathscr{H}_{\mu}$.

Suppose now that $\alpha \in \mathscr{D}^{\prime}(G)$ satisfies $0 \ll \alpha \ll \mu$. Then $I_{\alpha} \supseteqq I_{\mu}$, and there exists a unique self-adjoint operator $A$ on $\mathscr{H}_{\mu}$ such that

$$
(A \tilde{\phi}, \tilde{\psi})_{\mu}=\alpha\left(\psi^{*} * \phi\right) \text {. }
$$

The operator $A$ obviously satisfies

$$
\begin{gathered}
0 \leqq A \leqq I \\
L_{x}^{\mu} A=A L_{x}^{\mu},
\end{gathered}
$$

since the Hermitian form $\alpha\left(\phi^{*} * \phi\right)$ is nonnegative, bounded by $(\tilde{\phi}, \tilde{\phi})_{\mu}=$ $\|\tilde{\phi}\|_{\mu}^{2}$, and invariant under left translations by $G$. It was asserted (without proof) by Effros and Hahn in $[4, \S 4$ ] that when $\mu$ is a measure, then every operator $A$ satisfying (1.2) and (1.3) is given by formula (1.1), where $\alpha$ is a positive-definite measure. Unfortunately, this is false in general, as shown by the following example:

THEOREM 1. There is a positive-definite measure $\mu$ on the group $G=\mathrm{SL}(2, \boldsymbol{R})$ such that:

(i) The only measures $\alpha$ satisfying $0 \ll \alpha \ll \mu$ are the measures $c \mu, c \in[0,1]$. 
(ii) The representation $L^{\mu}$ of $G$ defined by $\mu$ is reducible.

If we allow positive-definite distributions in formula (1.1), however, then we obtain all intertwining operators, as follows:

Theorem 2. Let $G$ be a Lie group, and let $\mu$ be a positivedefinite distribution on $G$. Suppose $A$ is an operator on $\mathscr{H}_{\mu}$ satisfying (1.2) and (1.3). Then there exists a unique positive-definite distribution $\alpha$ on $G$ such that (1.1) holds. Furthermore, the local order of $\alpha$ can be bounded in terms of the local order of $\mu$ and the dimension of $G$.

REMARKS 1. Theorems 1 and 2 show that the cone of positivedefinite measures on $\mathrm{SL}(2, \boldsymbol{R})$ is not a face of the cone of positive-definite distributions.

2. For a study of unbounded intertwining operators, cf. [9].

3. In case $\mu$ is a positive-definite measure, then the distribution $\alpha$ in Theorem 2 has finite global order at most $2(\operatorname{dim} G+1)$.

A sequence $\left\{\phi_{n}\right\} \subset \mathscr{D}(G)$ will be called a $\delta$-sequence if $\phi_{n}(x) \geqq 0$, $\lim _{n} \int_{G} \phi_{n}(x) d x=1$, and $\operatorname{Supp}\left(\phi_{n}\right) \rightarrow\{1\}$ as $n \rightarrow \infty$. Any $\delta$-sequence is an approximate identity under convolution, of course.

CoROLlaRY. Let $\left\{\dot{\phi}_{n}\right\}$ be a delta sequence, and set $w_{n}=\phi_{n}^{*} * \phi_{n}$. Then the vector $\xi=\Sigma \lambda_{n} \widetilde{w}_{n}$ will be a cyclic vector for the representation $L^{\mu}$, provided $\lambda_{n}>0$ and $\lambda_{n} \longrightarrow 0$ sufficiently fast as $n \rightarrow \infty$.

2. Proof of Theorem 1. Let $G=\mathrm{SL}(2, \boldsymbol{R})$ in this section. We distinguish two closed subgroups of $G$ : the subgroup $B$ consisting of all matrices $b=\left(\begin{array}{ll}s & t \\ 0 & s^{-1}\end{array}\right)$, with $s, t$ real, $s \neq 0$, and the subgroup $V$ consisting of all matrices $v=\left(\begin{array}{ll}1 & 0 \\ x & 1\end{array}\right), x$ real. One has $B \cap V=\{1\}$, while $V \cdot B$ consists of all unimodular matrices $\left(\begin{array}{ll}a & b \\ c & d\end{array}\right)$, such that $a \neq 0$. The map $v, b \rightarrow v \cdot b$ is a diffeomorphism from $V \times B$ to the open subset $V \cdot B$ of $G$. Let $d v$ and $d b$ be left Haar measures on $V$ and $B$, respectively, and let $\Delta_{B}$ be the modular function of $B$. Left Haar measure $d x$ on $G$ is then given by the formula

$$
\int_{G} f(x) d x=\int_{V} \int_{B} f(v b) \Delta_{B}\left(b^{-1}\right) d b d v=\int_{B} \int_{V} f(b v) d b d v
$$


[2, Chap. VII, §3, Proposition 6].

Suppose that $p$ is a unitary character of $B$. Then $p(b) d b$ is a positive-definite measure on $B$, and the measure $\mu$ on $G$ defined by

$$
\int_{G} f(x) d \mu(x)=\int_{B} f(b) \Delta_{B}(b)^{-1 / 2} p(b) d b
$$

is positive-definite [1]. As in $\S 1$, we denote by $L^{\mu}$ the corresponding representation of $G$ on $\mathscr{H}_{\mu}$. The representation $L^{\mu}$ is equivalent to the "principal series" representation of $G$ induced from the one-dimensional representation $p$ of $B$. Using the integration formula (2.1), we can identify the representation space $\mathscr{H}_{\mu}$ with $L_{2}(V, d v)$. (This gives the so-called "non-compact picture" for the principal series [8].) Indeed, if $\phi, \psi \in \mathscr{D}(G)$, then an easy calculation using (2.1) shows that

$$
(\tilde{\phi}, \widetilde{\psi})_{\mu}=\int_{V} \varepsilon(\phi) \overline{\varepsilon(\psi)} d v
$$

where

$$
\varepsilon(\phi)(v)=\int_{B} \phi(v b) \Delta_{B}(b)^{-1 / 2} p(b) d b .
$$

The restriction of $L^{\mu}$ to the subgroup $V$ becomes simply the left regular representation of $V$ in this picture.

Lemma 1. Let $A$ be a bounded operator on $L_{2}(V)$ which commutes with left translations by $V$, and suppose that there exists a Radon measure $\alpha$ on $G$ such that

$$
(A \varepsilon(\phi), \varepsilon(\psi))_{L_{2}(V)}=\alpha\left(\psi^{*} * \phi\right)
$$

for all $\phi, \psi \in \mathscr{D}(G)$. Then there is a Radon measure $\nu$ on $V$ such that $A f=f * \nu$, for $f \in \mathscr{D}(V)$.

Proof. Since $A$ is translation invariant, it is enough to establish an estimate

$$
|(A f)(1)| \leqq C_{K}\|f\|_{\infty},
$$

for all $f \in \mathscr{D}(V)$ supported on an arbitrary compact set $K \subset V\left(\|f\|_{\infty}\right.$ denoting the sup norm). Let $\mathscr{C}^{\infty}(V)$ be the space of $C^{\infty}$ vectors for the left regular representation of $V$. By Sobolev's lemma, $\mathscr{H}^{\infty}(V) \subset$ $C^{\infty}(V)$, and $A$ leaves the space $\mathscr{H}^{\infty}(V)$ invariant. Hence, $A \varepsilon(\phi)$ is a $C^{\infty}$ function for every $\phi \in \mathscr{D}(G)$.

If $f \in \mathscr{D}(V)$ and $g \in \mathscr{D}(B)$, write $f \otimes g$ for the function $f(v) g(b)$. Via the map $v, b \rightarrow v b$ we may consider $f \otimes g$ as an element of $\mathscr{D}(G)$. Then $\varepsilon(f \otimes g)=\lambda_{g} f$, where $\lambda_{g}=\int_{B} g(b) \Delta_{B}(b)^{-1 / 2} p(b) d b$. In particular, 
if $\left\{f_{n}\right\}$ and $\left\{g_{n}\right\}$ are $\delta$-sequence in $\mathscr{D}(V)$ and $\mathscr{D}(B)$ respectively, then $\lambda_{g_{n}} \rightarrow 1$ as $n \rightarrow \infty$ and $f_{n} \otimes g_{n}$ is a $\delta$-sequence on $G$ (by the integration formula (2.1)). Hence, we deduce from (2.2) that

$$
A \varepsilon(\phi)(1)=\alpha(\phi)
$$

for all $\phi \in \mathscr{D}(G)$. Fix $g \in \mathscr{D}(B)$ such that $\lambda_{g}=1$. Then for any $f \in$ $\mathscr{D}(V)$ we have $f=\varepsilon(f \otimes g)$, and hence

$$
(A f)(1)=\alpha(f \otimes g) \text {. }
$$

Since $\alpha$ is a Radon measure, the right side of (2.4) satisfies (2.3), which proves the lemma. (In fact, $\nu$ is the measure $f \rightarrow \alpha(f \otimes g)$.)

Completion of proof of Theorem 1. Now take for $p$ the character $p(b)=\operatorname{sgn}(s)$, when $b=\left(\begin{array}{ll}s & t \\ 0 & s^{-1}\end{array}\right)$. Then it is known [8] that the induced representation $L^{\mu}$ in this case splits into two parts, and when $\mathscr{H}_{\mu}$ is realized as $L_{2}(V)$, then any nontrivial intertwining operator is a scalar multiple of the classical Hilbert transform

$$
A f(x)=\lim _{\delta \rightarrow 0} \frac{1}{\pi} \int_{|y|>\delta} f(x-y) y^{-1} d y .
$$

(We identify $V$ with $R$ via the map $x \rightarrow\left(\begin{array}{ll}1 & 0 \\ x & 1\end{array}\right)$ )

The Hilbert transform does not satisfy estimate (2.3). For example, if

$$
f_{n}(x)=\phi(x) \sum_{k=2}^{n} \frac{\sin (k x)}{k \log k},
$$

where $\phi \in \mathscr{D}(\boldsymbol{R})$ is fixed with $\phi(x)=1$ for $|x| \leqq 1$, then $\operatorname{Supp}\left(f_{n}\right) \leqq$ Supp $(\phi)$ and $\sup _{n}\left\|f_{n}\right\|_{\infty}<\infty$ [11, p. 182].

On the other hand,

$$
A f_{n}(0)=\sum_{k=2}^{n} c_{k}(k \log k)^{-1}+O(1)
$$

as $n \rightarrow \infty$, where

$$
c_{k}=\frac{1}{\pi} \int_{-1}^{1} x^{-1} \sin (k x) d x
$$

Since $c_{k} \rightarrow 1$ as $k \rightarrow \infty$, and since $\Sigma(k \log k)^{-1}=+\infty$, it follows that

$$
\sup _{n}\left|A f_{n}(0)\right|=\infty \text {. }
$$

3. Proof of Theorem 2 and Corollary. Let $G$ be an arbitrary Lie group (assumed countable at infinity), and let $\mu$ be a given positive- 
definite distribution on $G$. If we set $\|\phi\|_{\mu}=\mu\left(\phi^{*} * \phi\right)^{1 / 2}$, then $\phi \rightarrow\|\phi\|_{\mu}$ is a continuous seminorm on $\mathscr{D}(G)$. Suppose now that $A$ is a bounded operator on the representation space $\mathscr{H}_{\mu}$. We may associate with $A$ a bilinear form $B_{A}$ on $\mathscr{D}(G)$ by the formula

$$
B_{A}(\psi, \phi)=(A \tilde{\phi}, \widetilde{J \psi})_{\mu} \text {. }
$$

Here $\phi \rightarrow \tilde{\phi}$ is the canonical map from $\mathscr{D}(G)$ into $\mathscr{H}_{\mu}$ as in $\S 1$, and $J \dot{\phi}=\bar{\phi}$ (complex conjugate). By the Schwarz inequality and the boundedness of $A$ we see that

$$
\left|B_{A}(\psi, \phi)\right| \leqq\|A\|\|\phi\|_{\mu}\|J \psi\|_{\mu} \text {. }
$$

Clearly, $\psi \rightarrow\|J \psi\|_{\mu}$ is also a continuous seminorm on $\mathscr{D}(G)$. Although $\|J \psi\|_{\mu}$ need not be bounded in terms of $\|\psi\|_{\mu}$, nevertheless, the local order of this seminorm is the same as the local order of $\|\cdot\|_{\mu}$. (If $K \subset G$ is a compact set and $\rho$ is a continuous seminorm on $\mathscr{D}(G)$, we say that $\rho$ has order $\leqq r$ on $K$ if there is a finite set of differential operators $\left\{D_{j}\right\}$ on $G$ each of order $\leqq r$, such that $\rho(\phi) \leqq \max _{j}\left\|D_{j} \phi\right\|_{\infty}$ for all $\phi$ with $\operatorname{Supp}(\phi) \subseteq K$.)

The main analytic fact we need is the following version of the "kernel theorem" for continuous bilinear forms:

Lemma 2. Suppose $B$ is a bilinear form on $\mathscr{D}(G)$, and $\rho_{1}, \rho_{2}$ are continuous seminorms on $\mathscr{D}(G)$ such that

$$
|B(\phi, \psi)| \leqq \rho_{1}(\phi) \rho_{2}(\psi)
$$

Then there is a distribution $T$ on $G \times G$ such that

$$
B(\phi, \psi)=T(\phi \otimes \psi) \text {. }
$$

Furthermore, if $K_{1}$ and $K_{2}$ are compact subsets of $G$, and if $\rho_{j}$ has order $\leqq r_{j}$ on $K_{j}(j=1,2)$, then $T$ has order $\leqq r_{1}+r_{2}+2(\operatorname{dim} G+1)$ on any compact set $M \subset$ Interior $\left(K_{1} \times K_{2}\right)$.

Proof. Since multiplication by a $C^{\infty}$ function is an operator of order zero, we may use a partition of unity and local coordinates to reduce the problem to a local one in $\boldsymbol{R}^{d}, d=\operatorname{dim} G$, such that $K_{j}=$ $\{|x| \leqq 2\} \subseteq \boldsymbol{R}^{d}$ and $M=\{(x, y) ;|x| \leqq 1,|y| \leqq 1\} \subseteq \boldsymbol{R}^{d} \times \boldsymbol{R}^{d}$.

Let $\phi_{0} \in \mathscr{D}\left(\boldsymbol{R}^{d}\right)$ satisfy $\phi_{0}=1$ on $\{|x| \leqq 1\}$ and $\operatorname{Supp}\left(\phi_{0}\right) \subseteq K_{1}$. Set $e_{n}(x)=\phi_{0}(x) e^{i n \cdot x}$, where $n \in N^{d}$ and $n \cdot x=n_{1} x_{1}+\cdots+n_{d} x_{d}$. Then if $D$ is a differential operator of order $r$, one has $\left\|D e_{n}\right\|_{\infty} \leqq C(1+|n|)^{r}$. Hence, the a priori estimate (3.3) implies that for some constant $C>0$,

$$
\left|B\left(e_{m}, e_{n}\right)\right| \leqq C(1+|m|)^{r_{1}}(1+|n|)^{r_{2}}
$$

for all $m, n \in N^{d}$. 
Suppose now that $f$ is a $C^{\infty}$ function on $\boldsymbol{R}^{d} \times \boldsymbol{R}^{d}$ with $\operatorname{Supp}(f) \cong$ $M$. Then the Fourier series of $f$ can be written as

$$
f(x, y)=\sum_{m, n} \hat{f}(m, n) e_{m}(x) e_{n}(y),
$$

where $\{\hat{f}(m, n)\}$ are the Fourier coefficients of $f$. Define

$$
T(f)=\sum_{m, n} \hat{f}(m, n) B\left(e_{m}, e_{n}\right) \text {. }
$$

The series (3.5) is absolutely convergent, and by (3.4) we have the estimate

$$
|T(f)| \leqq C_{1} \sup _{m, n}\left\{|\hat{f}(m, n)|(1+|m|)^{r_{1}+d+1}(1+|n|)^{r_{2}+d+1}\right\},
$$

where $C_{1}=C \sum_{m, n}(1+|m|)^{-d-1}(1+|n|)^{-d-1}<\infty$. Since the right side of (3.6) is a seminorm of order $r_{1}+r_{2}+2 d+2$ on $M$, this proves the lemma.

Completion of proof of Theorem 2. Suppose now that the operator $A$ in formula (3.1) commutes with the representation $L^{\mu}$. Then the distribution $T$ on $G \times G$ such that $B_{A}(\phi, \psi)=T(\phi \otimes \psi)$, which was constructed in Lemma 2, satisfies for all $z \in G$,

$$
T\left(\delta_{z} f\right)=T(f), \quad f \in \mathscr{D}(G \times G),
$$

where $\delta_{z} f(x, y)=f\left(z^{-1} x, z^{-1} y\right)$.

The structure of distributions satisfying (3.7) was determined by Bruhat [3, Prop. 3.3]. Let $c$ denote the distribution on $G$ determined by left Haar measure, and let $\Phi: G \times G \rightarrow G \times G$ be the map $\Phi(x, y)=$ $(x, x y)$. Then (3.7) forces $T$ to have the form

$$
T(f)=(\iota \otimes \alpha)(f \circ \Phi),
$$

where $\alpha$ is a distribution on $G$. Symbolically,

$$
T(f)=\iint f(x, x y) d x d \alpha(y) \text {. }
$$

In particular, if $\phi, \psi \in \mathscr{D}(G)$, then

$$
\begin{aligned}
(A \tilde{\phi}, \tilde{\psi})_{\mu} & =T(J \psi \otimes \phi) \\
& =\iint \overline{\psi(x)} \phi(x y) d x d \alpha(y) \\
& =\alpha\left(\psi^{*} * \phi\right) .
\end{aligned}
$$

Hence, $\alpha$ serves to represent the intertwining operator $A$, and is obviously positive-definite if $A \geqq 0$. Since $\Phi$ is a diffeomorphism, the order of $\iota \otimes \alpha$ on a compact set $M \subset G \times G$ is the same as the order of $T$ on $\Phi^{-1}(M)$. By Lemma 2 and inequality (3.2), the local order 
of $\iota \otimes \alpha$ (and, hence, the local order of $\alpha$ ) can, therefore, be bounded in terms of the local order of $\mu$ and the dimension of $G$, as claimed.

Proof of Corollary. Using Theorem 2, we are able to rehabilitate the attempted proof of cyclicity in [7]. Given a $\delta$-sequence $\left\{\psi_{n}\right\}$ on $G$, let $K \subset G$ be a compact set such that $K=K^{-1}$ and $\operatorname{Supp}\left(\psi_{n}\right) \subseteq K$ for all $n$. Since $\|\psi\|_{\mu}$ is a continuous seminorm on $\mathscr{D}(G)$, there are right-invariant differential operators $D_{1}, \cdots, D_{r}$ on $G$ such that

$$
\|\psi\|_{\mu} \leqq \max _{j}\left\|D_{j} \psi\right\|_{\infty}
$$

for all $\psi$ supported on the set $K^{2}$.

Now set $w_{n}=\psi_{n}^{*} * \psi_{n}$, and let $\left\{\lambda_{n}\right\}$ be any sequence such that $\lambda_{n}>0$ and

$$
\sum_{n} \lambda_{n} \max _{j}\left\|D_{j} \psi_{n}\right\|_{\infty}^{2}<\infty \text {. }
$$

The series $\xi=\sum \lambda_{n} \widetilde{w}_{n}$ then converges absolutely in $\mathscr{C l}_{\mu}$ (since $\left\|w_{n}\right\|_{\mu} \leqq$ $\left.\left\|\psi_{n}\right\|_{\psi^{2}}^{2}\right)$. Let $\mathscr{N}$ be the $G$-cyclic subspace generated by $\xi$, and let $A$ be the projection onto $\mathscr{N}^{\perp}$. Since $A \xi=0$, we have $\sum \lambda_{n}\left(A \widetilde{w}_{n}, \widetilde{\phi}\right)_{\mu}=$ 0 for all $\phi \in \mathscr{D}(G)$. But $\widetilde{\phi * \psi}=L_{\mu}(\phi) \widetilde{\psi}$, where $L_{\mu}(f)=\int f(x) L_{\mu}(x) d x$ is the integrated form of the representation. Since $A$ commutes with $L_{\mu}$, this gives $\left(A \widetilde{w}_{n}, \tilde{\phi}\right)_{\mu}=\left(A \widetilde{\psi}_{n}, \widetilde{\psi_{n} * \phi}\right)_{\mu}$. Thus taking $\dot{\phi}=\psi_{\psi_{k}}$ and letting $k \rightarrow \infty$, we see that

$$
\lim _{k \rightarrow \infty}\left(A \widetilde{w}_{n}, \widetilde{\psi}_{k}\right)_{\mu}=\left(A \widetilde{\psi}_{n}, \widetilde{\psi}_{n}\right)_{\mu}
$$

(note that $\phi \rightarrow \tilde{\phi}$ is continuous from $\mathscr{D}(G)$ to $\mathscr{C}_{\mu}$ ). Furthermore, by the Schwartz inequality, the boundedness of $A$, and the calculation just made, we have the estimate

$$
\begin{aligned}
\left|\left(A \widetilde{w}_{n}, \widetilde{\psi}_{k}\right)_{\mu}\right| & \leqq\left\|\psi_{n}\right\|_{\mu}\left\|\psi_{n} * \psi_{k}\right\|_{\mu} \\
& \leqq C \max _{j}\left\|D_{j} \psi_{n}\right\|_{\infty}^{2} .
\end{aligned}
$$

(Here we have used estimate (3.8), the right-invariance of $D_{j}$, and the inequality $\|f * g\|_{\infty} \leqq\|f\|_{\infty}\|g\|_{L_{1}}$. . Thus we may apply the dominated convergence theorem to conclude from (3.9) and (3.10) that $\sum \lambda_{n}\left(A \widetilde{\psi}_{n}, \widetilde{\psi}_{n}\right)_{\mu}=0$. But $\lambda_{n}>0$ and $A \geqq 0$, so in fact $\left(A \widetilde{\psi}_{n}, \widetilde{\psi}_{n}\right)_{\mu}=$ 0 for all $n$. (So far we have simply followed the line of proof of [7], replacing uniform convergence of the series $\sum \lambda_{n} w_{n}$ by the stronger condition (3.9), in return for allowing $\mu$ which are distributions rather than measures.) Finally let $\alpha$ be the positive-definite distribution on $G$ representing $A$, which exists by Theorem 2. Then $\alpha\left(\psi_{n}^{* *} \psi_{n}\right)=0$ for all $n$. By the Schwarz inequality, this implies that $\alpha\left(\phi * \psi_{n}\right)=0$ for all $\phi \in \mathscr{D}(G)$ and all $n$. Letting $n \rightarrow \infty$, we conclude that $\alpha=0$. 


\section{REFERENCES}

1. R. J. Blattner, Positive-definite measures, Proc. Amer. Math. Soc., 14 (1963), 423428.

2. N. Bourbaki, Intégration, Chap. VII-VIII (Act. Sc. Ind., No. 1306, Hermann, Paris, 1963).

3. F. Bruhat, Sur les représentations induites des groupes de Lie, Bull. Soc. Math., France, 84 (1956), 97-205.

4. E. G. Effros and F. Hahn, Locally compact transformation groups and $C^{*}$ algebras, Mem. Amer. Math. Soc., No. 75, 1967.

5. I. M. Gelfand and D. Raikov, Irreducible unitary representations of locally compact groups, Mat. Sbornik, 13 (1943), 301-316.

6. F. Greenleaf and M. Moskowitz, Cyclic vectors for representations of locally compact groups, Math. Ann., 190 (1970), 265-288.

7. A. Hulanicki and T. Pytlik, Cyclic vectors of induced representations, Proc. Amer. Math. Soc., 31 (1972), 633-634.

8. A. W. Knapp and E. M. Stein, Intertwining operators for semisimple groups, Ann. of Math., (2) 93 (1971), 489-578.

9. Neils Skovhus Poulsen, On $C^{\infty}$ vectors and intertwining bilinear forms for representations of Lie groups, J. Functional Analysis, 9 (1972), 87-120.

10. F. Treves, Topological Vector Spaces, Distributions, and Kernels, Academic Press, New York, 1967.

11. A. Zygmund, Trigonometric Series, Vol. I, 2nd. Ed., Cambridge Univ. Press, Cambridge, 1968.

Received June 6, 1972 and in revised form November 3, 1972. Partially supported by NSF Grant GP 33567.

RUTGERS UNIVERSITY 



\section{PACIFIC JOURNAL OF MATHEMATICS}

\section{EDITORS}

RICHARD ARENS (Managing Editor)

University of California

Los Angeles, California 90024

R. A. Beaumont

University of Washington

Seattle, Washington 98105
J. DUGUNDJI*

Department of Mathematics

University of Southern California

Los Angeles, California 90007

D. Gilbarg and J. Milgram

Stanford University

Stanford, California 94305

\section{ASSOCIATE EDITORS}
E. F. BECKENBACH
B. H. NeumanN
F. WOLF
K. YosHIDA

\section{SUPPORTING INSTITUTIONS}

UNIVERSITY OF BRITISH COLUMBIA
CALIFORNIA INSTITUTE OF TECHNOLOGY
UNIVERSITY OF CALIFORNIA
MONTANA STATE UNIVERSITY
UNIVERSITY OF NEVADA
NEW MEXICO STATE UNIVERSITY
OREGON STATE UNIVERSITY
UNIVERSITY OF OREGON
OSAKA UNIVERSITY

UNIVERSITY OF BRITISH COLUMBIA

UNIVERSITY OF CALIFORNIA

MONTANA STATE UNIVERSITY

UNIVERSITY OF NEVADA

OREGON STATE UNIVERSITY

OSAKA UNIVERSITY
UNIVERSITY OF SOUTHERN CALIFORNIA

STANFORD UNIVERSITY

UNIVERSITY OF TOKYO

UNIVERSITY OF UTAH

WASHINGTON STATE UNIVERSITY

UNIVERSITY OF WASHINGTON

\section{AMERICAN MATHEMATICAL SOCIETY} NAVAL WEAPONS CENTER

* C. R. DePrima California Institute of Technology, Pasadena, CA 91109, will replace J. Dugundji until August 1974. 


\section{Pacific Journal of Mathematics}

\section{Vol. 48, No. $1 \quad$ March, 1973}

Jan Aarts and David John Lutzer, Pseudo-completeness and the product of Baire

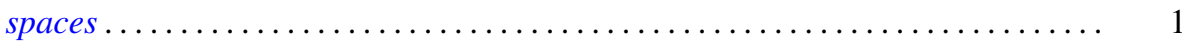

Gordon Owen Berg, Metric characterizations of Euclidean spaces ............ 11

Ajit Kaur Chilana, The space of bounded sequences with the mixed topology ..... . 29

Philip Throop Church and James Timourian, Differentiable open maps of

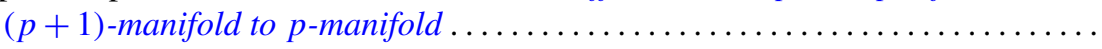

P. D. T. A. Elliott, On additive functions whose limiting distributions possess a finite

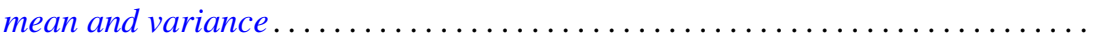

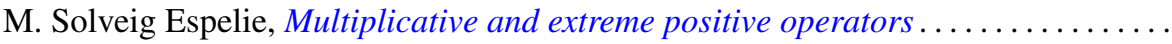

Jacques A. Ferland, Domains of negativity and application to generalized convexity

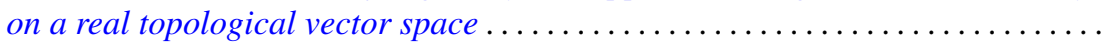

Michael Benton Freeman and Reese Harvey, A compact set that is locally holomorphically convex but not holomorphically convex ...............

Roe William Goodman, Positive-definite distributions and intertwining

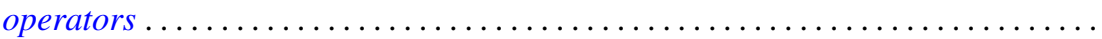

Elliot Charles Gootman, The type of some $C^{*}$ and $W^{*}$-algebras associated with

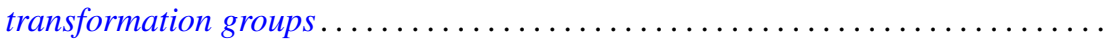

David Charles Haddad, Angular limits of locally finitely valent holomorphic

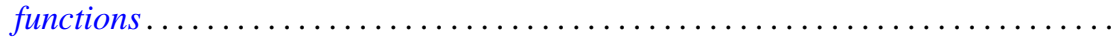

William Buhmann Johnson, On quasi-complements .

William M. Kantor, On 2-transitive collineation groups of finite projective spaces...

Joachim Lambek and Gerhard O. Michler, Completions and classical localizations of right Noetherian rings

Kenneth Lamar Lange, Borel sets of probability measures ......

David Lowell Lovelady, Product integrals for an ordinary differential equation in a Banach space

Jorge Martinez, A hom-functor for lattice-ordered groups .........

W. K. Mason, Weakly almost periodic homeomorphisms of the two sphere ....

Anthony G. Mucci, Limits for martingale-like sequences .......

Eugene Michael Norris, Relationally induced semigroups ...

Arthur E. Olson, A comparison of c-density and $k$-density ......

Donald Steven Passman, On the semisimplicity of group rings of linear groups.

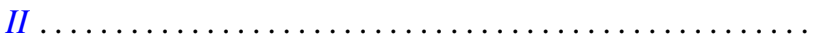

Charles Radin, Ergodicity in von Neumann algebras .

P. Rosenthal, On the singularities of the function generated by the Bergman operator of the second kind.

Arthur Argyle Sagle and J. R. Schumi, Multiplications on homogeneous spaces,

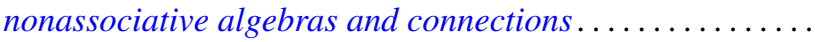

Leo Sario and Cecilia Wang, Existence of Dirichlet finite biharmonic functions on

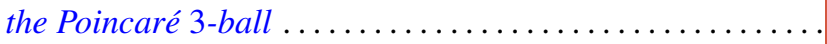

Ramachandran Subramanian, On a generalization of martingales due to Blake ..

Bui An Ton, On strongly nonlinear elliptic variational inequalities.

Seth Warner, A topological characterization of complete, discretely valued

fields. 